Supporting Information for

\title{
Microrheological Study of Physical Gelation in Living Polymeric Networks
}

\author{
Tetsuharu Narita ${ }^{\dagger}$ and Tsutomu Indei $i^{*, \ddagger}$ \\ $\dagger$ Laboratoire PPMD-SIMM, UPMC-ESPCI ParisTech-CNRS, UMR7615, 10 rue \\ Vauquelin, 75005 Paris, France \\ $\ddagger$ Department of Chemical and Biological Engineering, and Center for Molecular Study of \\ Condensed Soft Matter, Illinois Institute of Technology, 3440 S. Dearborn St., Suite 150, \\ Chicago, Illinois 60616, U.S.A. \\ E-mail: tindei@iit.edu
}

\section{Mean-field theory of thermoreversible gelation}

In an equilibrium mean-field theory developed by Ishida and Tanaka in 1997, gel point is predicted as ${ }^{61}$

$$
C_{\mathrm{p}}^{*}=\text { const. } \times \frac{f-1}{f(f-2)^{2}},
$$

where $f$ is the functionality or the number of stickers per polymer. A prefactor is a constant independent of $f$. Shortly after that, Semenov and Rubinstein derived the same result. ${ }^{62}$ Both theories assume (i) monodispersity of the functionality, 


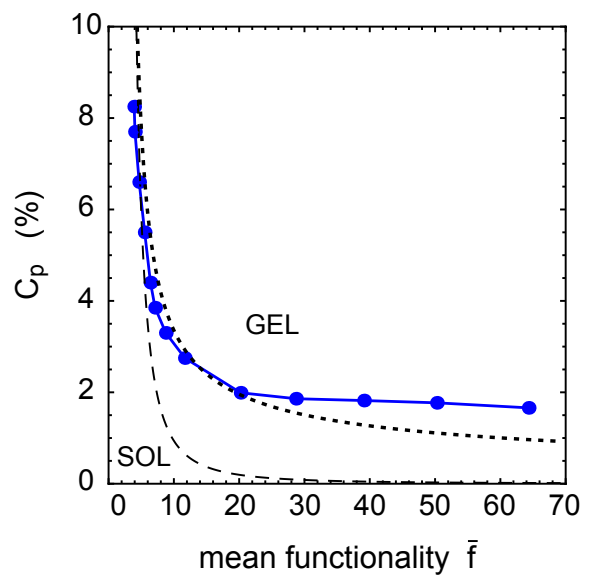

Figure S1: Sol-gel phase diagram for PVA-borax aqueous solution/gel. Symbols: experimental data (from Fig.8 of the paper). Dashed line is from Eq.(S1) whereas dotted line is from Eq.(S2).

(ii) association between free (or open) stickers, (iii) pairwise association, and (iv) no loop-formation in pregel regime.

In Fig.S1, experimental result presented in Fig.8 is compared with the prediction from Eq.(S1) where the mean value of the functionality $\bar{f}$ is used for $f$. We put const. $=66.8$ so that the asymptotic value of $\bar{f}$ at high $C_{\mathrm{p}}(\geq 4.4 \%)$ fits with the experimental value on average. We can see that the overall feature of the experimental sol-gel boundary agrees with the theoretical curve from Eq.(S1). But there is a large discrepancy at low PVA concentrations. That is, the model predicts gelation at a lower polymer concentration for a given mean functionality than the experimental result.

Now we briefly discuss the validity of the assumptions (i)-(iv) of the theory when it is applied to the present PVA-borax system. The first assumption is not precisely consistent with the present system because the functionality is polydisperse since the molecular weight of the PVA chain is polydisperse and the number of the diol complexes on the chain fluctuates with time. In spite of that, the average value of the functionality can be used reasonably. The second assumption is acceptable for the present system despite the fact that the transient crosslinkers 
(borate ions) are not fixed to the chain permanently. The third assumption is reasonable because any monomer can become a sticker by the monodiol complexation with a borate ion on a one-to-one base. The last one is a typical assumption employed for convenience in conventional theories. However the absence of the loops is not realistic especially at low polymer concentrations.

\section{Strong bond-strength limit}

The assumption (iv) may be removed in part by making use of the SemenovRubinstein's argument about renormalization of the bond strength when the bond strength is very high. ${ }^{62}$ They assumed that in the high bond strength limit, all nearest neighbor pairs of stickers along the chain are locally intra-associated at low polymer concentration. Large loops are not considered in this theory. Then the gel point is predicted as the following equation:

$$
C_{\mathrm{p}}^{*}=\text { const. } \times \frac{f^{1 / 2}(f-1)}{(f-2)^{2}} .
$$

We derived this equation naïvely by putting Eq.(2.20) into Eq.(3.8) of Ref. ${ }^{62}$. We assumed that the chain is Gaussian (the exponent 1/2 is originated from this assumption). As before, to fit Eq.(S2) with the experimental curve, we adjust the constant prefactor (which is different from the one in Eq.(S1)) so that the asymptotic value of $\bar{f}$ at high $C_{\mathrm{p}}$ coincides with that of Eq.(S1).

We can see from Fig.S1 that the theoretical curve from Eq.(S2) agrees with experimental data better than the curve from Eq.(S1). It indicates that the inclusion of the loop formation in the theory changes the curve in right direction. Still there is a discrepancy between the theory and the experiment at low PVA concentrations. Probably this is because at low PVA concentration not all borate ions make complexation. As previously shown, ${ }^{31}$ the estimated amount of efficient crosslinkers is much lower than the amount of the crosslinkers in the system, suggesting that a certain amount of crosslinkers are in part inefficiently 
crosslinking (loop/cycle) and in part not crosslinking at all (monodiol binding or no binding).

In addition, differently from the assumption (ii), open stickers (monodiol complexes) can connect with any monomer so that various types or topologies of intra-chain association are possible in the PVA-borax system, whereas the theory considers only the local loop formation by two nearest neighbor stickers. In order to describe this system more appropriately, change of the configurational entropy associated with the formation of all these types of the loops should be taken into account in the theory. We do not go into more detail here. 